\title{
A NOTE ON NORMALLY GENERATED LINE BUNDLES ON COMPACT RIEMANN SURFACES
}

\author{
By \\ Tatsuya Arakawa
}

\section{Introduction}

Let $X$ denote a compact Riemann surface of genus $g(X)>0$ and $L$ an ample line bundle on $X$.

DEFINITION 1. $L$ is said to be normally generated if, for each $n>0$, the natural map

$$
\operatorname{Sym}^{n} H^{0}(X, L) \rightarrow H^{0}\left(X, L^{n}\right)
$$

is surjective.

There are the following two sufficient conditions for line bundles on $X$ to be normally generated obtained by $\mathrm{H}$. H. Martens and D. Mumford, respectively:

THEOREM 1 (cf. [3]). The canononical bundle $K_{X}$ on $X$ is normally generated if and only if $X$ is nonhyperelliptic.

THEOREM 2 ([4]). If $\operatorname{deg} L \geq 2 g(X)+1$, then L/is normally generated.

On the other hand, Homma [2] classified all the normally generated line bundles on $X$ when the genus of $X$ is three.

THEOREM 3 ([2]). Supppose $g(X)=3$.

(i) If $X$ is hyperelliptic, then $L$ is normally generated if and only if $\operatorname{deg} L \geq 7$.

(ii) If $X$ is nonhyperelliptic, then $L$ is normally generated if and only $L$ satisfies one of the following conditions:

(a) $\operatorname{deg} L \geq 7$.

Received December 18, 1997

Revised May 1, 1998 
(b) $\operatorname{deg} L=6$ and $H^{0}\left(X, L \otimes K_{X}^{-1}\right)=0$.

(c) $L \cong K_{X}$.

Now let $\pi: X \rightarrow Y$ be a (possibly ramified) covering of compact Riemann surfaces and let $g(Y)(\geq 0)$ denote the genus of $Y$.

Problem. Classify ample line bundles on $Y$ such that the pull backs on $X$ are normally generated.

In this note, we will study this problem in the cases of $\pi$ being double coverings with small $g(X)$ or $g(Y)$. In $\S 2$, we will determine such line bundles on $Y$ when $g(X)=3$ and in $\S 3$, the cases of $Y$ being rational or elliptic Riemann surfaces are treated.

Before closing this section, let us recall some fundamental facts on double coverings (cf. [5]):

Lemma 1. Let $B$ denote the branch locus of the double covering $\pi: X \rightarrow Y$ on $Y$. Then there exists a line bundle $F$ on $Y$ with $2 F \cong B$ such that the following conditions hold:

(i) $X$ is embedded into $F$ and the projection of $F$ to $Y$ restricted on $X$ coincides with $\pi$.

(ii) The canonical bundle $K_{X}$ on $X$ is linearly equivalent to $\pi^{*}\left(K_{Y} \otimes F\right)$ where $K_{Y}$ is the canonical bundle on $Y$.

(iii) For any line bundle $L$ on $Y$, we have:

$$
\pi_{*} \mathcal{O}_{X}\left(\pi^{*} L\right) \cong \mathcal{O}_{Y}(L) \oplus \mathcal{O}_{Y}\left(L \otimes F^{-1}\right) .
$$

Corollary. Let $\pi: X \rightarrow Y$ be a double covering of compact Riemann surfaces. Then the induced homomorphism $\pi^{*}: \operatorname{Pic} Y \rightarrow \operatorname{Pic} X$ is injective.

Proof. Let $M$ be a line bundle on $Y$ such that the pull back $\pi^{*} M$ is trivial on $X$. Then we have $\operatorname{deg} M=0$ and $h^{0}\left(X, \pi^{*} M\right)=1$. Hence, by Lemma 1 (iii), we have $h^{0}(Y, M)=h^{0}\left(X, \pi^{*} M\right)-h^{0}\left(Y, M \otimes F^{-1}\right)=1$, that is, $M$ is also trivial on $Y$.

The author would like to express his gratitude to Professors Sampei Usui and Kazuhiro Konno for their helpful suggestions and encouragements. He also thanks to Professor Masayoshi Miyanishi for useful advises. Finally he thanks to the referee for pointing out several mistakes in the earlier version. 


\section{The cases of $g(X)=3$}

By Lemma 1 and Theorem 3, we can determine such line bundle $M$ on $Y$ as in Problem when $g(X)=3$ :

Since $g(X)>g(Y)$, we have $g(Y)=0,1$ or 2. If $g(Y)=0$, then $X$ is hyperelliptic. On the other hand, we have the following result of Farkas:

LEMMA 2 ([1]). Let $X \rightarrow Y$ be a double covering of compact Riemann surfaces with $g(X)=3$ and $g(Y)=2$. Then $X$ is hyperelliptic.

As a conclusion of Lemma 2 and Theroem 3 (i), we obtain the following result.

Proposition 1. Suppose $g(Y)=0$ or 2. Then $\pi^{*} M$ is normally generated if and only if $\operatorname{deg} M \geq 4$.

Now suppose $X$ is nonhyperelliptic. Then we have $g(Y)=1$ and hence, by Lemma 1 (ii), $K_{X} \cong \pi^{*} F$ and $\operatorname{deg} F=2$.

By Theorem 3 (ii), $\pi^{*} M$ is normally generated if $\operatorname{deg} M \geq 4$ and not normally generated if $\operatorname{deg} M=1$.

Suppose $\operatorname{deg} M=2$. Then $\pi^{*} M$ is normally generated if and only if $\pi^{*} M \cong \pi^{*} F$, that is, by the corollary to Lemma 1, $M \cong F$.

Suppose $\operatorname{deg} M=3$. Then, since $g(Y)=1$ and $\operatorname{deg} M \otimes F^{-1}=1$, we have

$$
h^{0}\left(X, \pi^{*} M \otimes K_{X}^{-1}\right)=h^{0}\left(Y, M \otimes F^{-1}\right)+h^{0}\left(Y, M \otimes F^{-2}\right)>0 .
$$

Consequently we get the following:

Proposition 2. Suppose $g(Y)=1$.

(i) If $X$ is hyperelliptic then $\pi^{*} M$ is normally generated if and only if $\operatorname{deg} M \geq 4$.

(ii) If $X$ is nonhyperelliptic then $\pi^{*} M$ is normally generated if and only if $\operatorname{deg} M \geq 4$ or $M \cong F$.

3. The cases of $g(X) \geq 4$ and $g(Y) \leq 1$

3.1. The cases of $g(Y)=0$

If $g(Y)=0$, then $\operatorname{deg} F=g(X)+1$ and hence, if $\operatorname{deg} M<g(X)+1$ for a line bundle $M$ on $Y$, we have

$$
H^{0}\left(X, \pi^{*} M\right) \cong H^{0}(Y, M)
$$


by Lemma 1 (iii), that is, each section in $H^{0}\left(X, \pi^{*} M\right)$ is the pull back of a section in $H^{0}(Y, M)$. But, for a sufficiently large $n$,

$$
H^{0}\left(X, \pi^{*} M^{n}\right) \neq H^{0}\left(Y, M^{n}\right)
$$

by Lemma 1 (iii) again. We therefore conclude that $\pi^{*} M$ is not normally generated in this case.

On the other hand, by Theorem $2, \pi^{*} M$ is normally generated if $\operatorname{deg} M \geq$ $g(X)+1$.

Consequently we have:

Proposition 3. Suppose $g(Y)=0$. Then $\pi^{*} M$ is normally generated if and only if $\operatorname{deg} M \geq g(X)+1$.

\subsection{The cases of $g(Y)=1$}

If $g(Y)=1$, then we have $\operatorname{deg} F=g(X)-1$ and $K_{X} \cong \pi^{*} F$. If moreover $g(X) \geq 4$, then $X$ is always nonhyperelliptic.

Now by the same arguments as in $\S 3.1$, we can conclude that, for a line bundle $M$ on $Y, \pi^{*} M$ is not normally generated if $H^{0}\left(X, M \otimes F^{-1}\right)=0$. Therefore if $\pi^{*} M$ is normally generated, then $\operatorname{deg} M \geq g(X)$ or $M \cong F$. On the other hand, by Theorems 1 and $2, \pi^{*} M$ is normally generated if $\operatorname{deg} M \geq$ $g(X)+1$ or $M \cong F$.

Now suppose $\operatorname{deg} M=g(X)$. By Lemma 1 (iii), we have

$$
H^{0}\left(X, \pi^{*} M\right) \cong H^{0}(Y, M) \oplus H^{0}\left(Y, M \otimes F^{-1}\right)
$$

and

$$
H^{0}\left(X, \pi^{*} M^{2}\right) \cong H^{0}\left(Y, M^{2}\right) \oplus H^{0}\left(Y, M^{2} \otimes F^{-1}\right) .
$$

But by the Riemann-Roch theorem, we have $h^{0}(Y, M)=g, h^{0}\left(Y, M \otimes F^{-1}\right)=1$ and $h^{0}\left(Y, M^{2} \otimes F^{-1}\right)=g+1$. Hence the natural map

$$
H^{0}(Y, M) \otimes H^{0}\left(Y, M \otimes F^{-1}\right) \rightarrow H^{0}\left(Y, M^{2} \otimes F^{-1}\right)
$$

is not surjective, and neither is

$$
H^{0}\left(X, \pi^{*} M\right) \otimes H^{0}\left(X, \pi^{*} M\right) \rightarrow H^{0}\left(X, \pi^{*} M^{2}\right) .
$$

Therefore we conclude that, in this case, $\pi^{*} M$ is not normally generated. 
Consequently we have:

Proposition 4. Suppose $g(X) \geq 4$ and $g(Y)=1$. Then, for a line bundle $M$ on $Y, \pi^{*} M$ is normally generated if and only if $\operatorname{deg} M \geq g(X)+1$ or $M \cong F$.

\title{
References
}

[1] Accola, R. M., Riemann surfaces with automorphism groups admitting partitions, Proc. Amer. Math. Soc. 21 (1969), 477-482.

[2] Homma, M., On projective normality and defining equations of a projective curve of genus 3, Tsukuba J. Math. 4 (1980), 269-279.

[ 3 ] Martens, H. H., Varieties of special divisors on a curve. II, J. Reine Angew. Math. 233 (1968), 89-100.

[4] Mumford, D., Varieties defined by quadratic equations, in Questions on Algebraic varieties, CIME (1970) 29-100.

[5] Persson, U., Double coverings and surfaces of general type, Springer Lecture notes in mathematics 687 (1978).

\author{
Department of Mathematics \\ Graduate School of Science \\ Osaka University \\ Toyonaka Osaka 560-0043 \\ Japan \\ E-mail adress: arakawa@math.sci.osaka-u.ac.jp
}

